\title{
A review of wrist pulse analysis.
}

\author{
Suguna GC*, Veerabhadrappa ST \\ Department of Electronics and Communication Engineering, JSS Academy of Technical Education, Bengaluru, \\ Karnataka, India
}

\begin{abstract}
In present scenario, the human health condition is being diagnosed by various advanced sophisticated equipments. Moreover, the process of diagnosis is becoming expensive and painful. In ancient Indian Ayurveda and Traditional Chinese Medicine (TCM), wrist pulse was used to diagnose the health status of the subject and to envision the root cause of a disease. According to Ayurveda, a person's health status depends on three essential components and the imbalance of a component is the symptom of a disease. Practitioner determines the unhealthiness of a subject, based on the wrist pulse parameters such as pulse strength, amplitude shape, vega, rhythm, pulse width and transition period. Evaluation of the vital information of the components depends exclusively upon the perception of the practitioner and varies from practitioner to practitioner based on his/her expertise or experience. No modern technique can determine the pathological condition of a subject. The paper presents a review of wrist pulse parameters for diseases like diabetics, hypertension, gastritis, etc.
\end{abstract}

Keywords: Wrist pulse, Vata, Pitta, Kapha.

Accepted on May 17, 2019

\section{Introduction}

In today's world, a human's health state is diagnosed with modern techniques which are expensive. In Ancient times, the health status of a subject under consideration is determined by an Ayurvedic physician by feeling palpation from three fingers placed on the radial artery for Vata, Pitta and Kapha components respectively and it's commonly known as "Nadi Vidya". Nadi Vidya is a simple, cost effective, painless, nonevasive approach to measure the health status of the subject using wrist pulse parameters and to determine the root cause for the same. According to Ayurveda, the human anatomy comprises of Ether, Air, Fire, Water and Earth that are manifested into Vata, Pitta and Kapha elements. Imbalance of Vata, Pitta and Kapha components leads to unhealthiness of a subject. The unhealthy state was determined based on the predominant Vata, Pitta, Kapha components [1].

Vata is a combination of Ether and Air which attributes to two main aspects, one of them being associated with movements within the body and mind, with the other being associated with communication. Vata have sub elements as prana, apana, samana, vyan and udana. Imbalance of Vata commonly affects the nerve, neck, abdomen, umbilicus, bladder and rectum disorders and observed in old age. The Vata component possesses certain distinct characteristics such as irregular rhythm patterns, 80-90 bpm, fast, feeble, cold with lighter weight. Pitta is a combination of Fire and Water which represents frog like attributes within the body such as digestion and metabolism. Pitta has sub elements as alochaka, bhrajaka, sadhaka, pachaka and ranjaka. Imbalance of Pitta alone results in vision, metabolic, digestive system disorders and is noticeable in middle age. Pitta component also possesses unique characteristics such as having regular rhythm patterns with prominent amplitude of the pulse, 70-80 bpm and is also associated with heat and moistness. Kapha is a combination of Earth and Water which governs the structure and fluids of the body. Water energy, Kapha have sub elements tarpaka, bodhaka, avalambaka, kledaka and shleshaka. Kapha variations is seen in subject such as, phlegmatic, sinus congestion, poor sense of taste and smell, lower back pain, respiratory, weight gain and are more prominent in childhood.

The characteristics of Kapha are regular rhythm patterns, 50-60 bpm, having slow and wavy movements [2-5]. Vata, Pitta and Kapha elements controls the mind, body, soul and spirit. Diagnosis of radial pulse is a unique technique in Ayurvedic science which reveals the existing health status of the subject and its root cause. Pulse wave is a pressure signal that could be recorded on foot, radial, brachial, carotid and femoral parts of the human system. Normally, pressure signals are measured on the wrist as they are convenient, easy and accurate to assess the strength of the pulse. The pressure signal manifests itself due to the contraction and relaxation of the heart muscles which build up pressure across the walls of arteries. Pressure signal contains vital information about physiological and pathological health state of the subject [5].

Ayurvedic practitioner feels the pulse by applying static pressure through index, middle and ring fingers to estimate the static and dynamic nature of Vata, Pitta and Kapha 
components. Practitioner examines several parameters such as rhythm, amplitude, depth, width and vega of a dynamic pulse to determine the health status of a subject. Variation in the pulse amplitude, duration, pulse wave velocity, blood volume and pulse rate are evaluated in time domain. Frequency and its harmonic frequencies, frequency shift, phase shifts, power, energy are measured and analyzed in frequency domain. Transition of Vata, Pitta and Kapha components in time domain and frequency domain analysis indicates many physiological and pathological characteristics of the human system. Wrist Pulse is a pressure signal possessing a frequency band less than $20 \mathrm{~Hz}$ for a healthy subject.

Superficial pressure on the left hand is used to detect disorders related to small intestine, gall bladder, urinary bladder whereas superficial pressure applied on the right hand helps to detect disorders related to large intestine, stomach, triple energizer. However, application of deep pressure applied on the left hand is used to determine disorders related to heart, liver and kidneys while applying the same pressure only on the right hand can be used to indicate several problems within the lungs, spleen and pericardium [6] and shown in Figure 1.

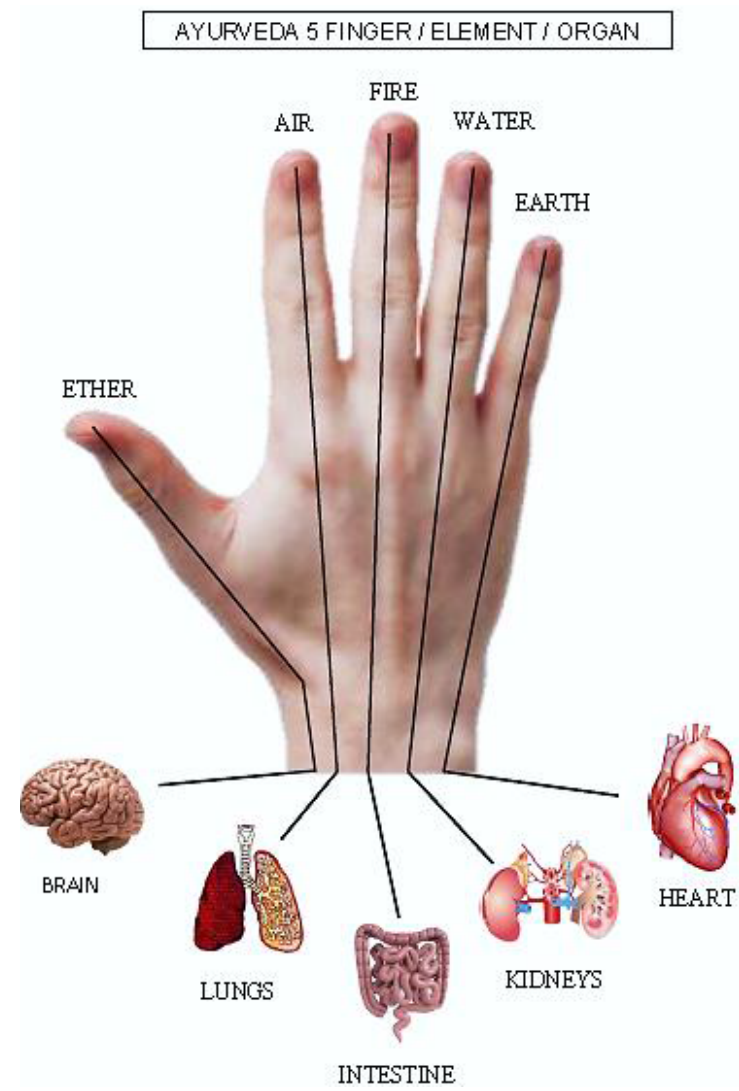

Figure 1. Organs related to fingers.

Figure 2 shows the typical pulse wave with percussion, tidal wave and dicrotic wave. Percussion wave having maxim amplitude occurs during ventricular contraction, pumping the blood into aorta. Percussion wave contains information about the heart rhythm and cardiac contraction. Tidal wave and Dicrotic wave occurs due to the relaxation of ventricular. Majority of the information about the arterial system lies in tidal and dicrotic waves which ultimately results in wrist pulse pattern [7].

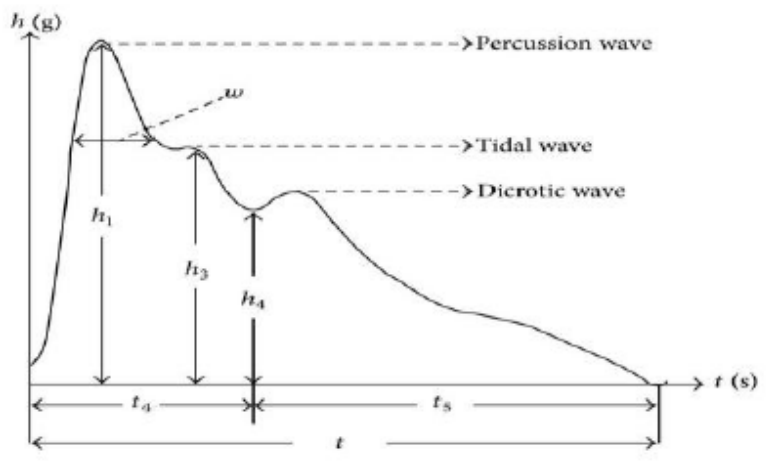

Figure 2. Pressure signal.

On the application of various levels of pressure on the radial pulse points, the three dynamic pressure components (Vata, Pitta and Kapha) can be obtained as shown in Figure 3.
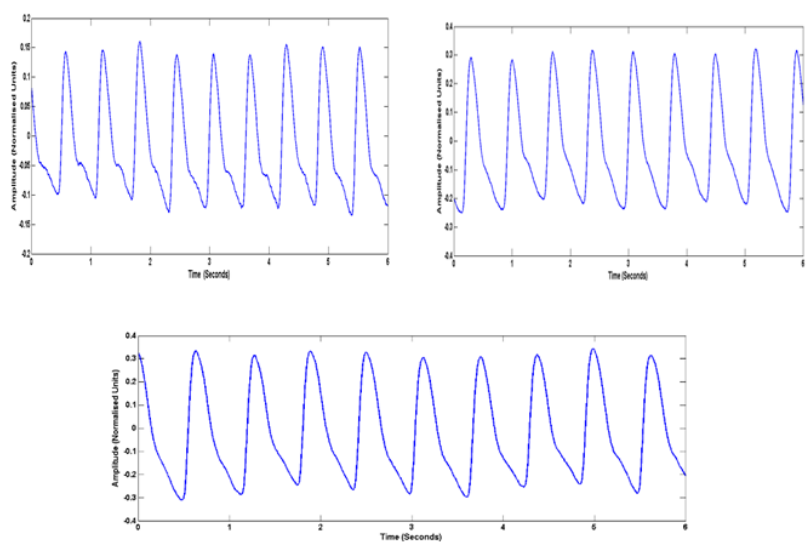

Figure 3. Wrist pulse signals (a) Vata, (b) Pitta and (c) Kapha Pulse.

This paper presents the use of various sensors for pulse signal analysis which includes time domain, frequency domain, and time-frequency domain and also looks into the case studies of various diseases.

\section{Radial pulse measuring system}

Sensors play a vital role to estimate the static and dynamic component of a wrist pulse. Several pulse measurement systems have emerged to measure the wrist pulse, based on acoustic, piezoelectric, piezoresistive, infrared, ultrasound, flexible MEMS sensors and biosensors.

Acoustic sensors: Acoustic sensors are based on piezoelectric material. The velocity and amplitude of an acoustic wave during propagation is affected due to changes in the propagation medium. Microphone, transducers are used to convert acoustic signal into electrical signal designed to capture the signal above $20 \mathrm{~Hz}$, but the pulse signal of a healthy subject has a frequency component below $20 \mathrm{~Hz}$ hence they are not suitable for acquiring the radial pulse. 
Optic sensors: Optical based measurement of pulse contains two components namely, a light emitting source and photo detector. Infra-red LED emits the light on to the subject which enters deeply into blood vessel and tissues. Backscattered light from the subject was captured by the photo detector. Backscattered light varies with the blood flow in the arteries result in wrist pulse. Radial pulses obtained from optic sensors indicate Pulse Wave Velocity, $\mathrm{PTT}, \mathrm{SPO}_{2}$ and heart rate. Merits of optical sensors are low cost, portable, high speed, high accuracy and precision. Hence they can be used in the measurement of heart rate and blood pressure. Optic sensors are not suitable for the study of dynamic characteristics of the radial pulse. In spatial distribution, pulse sensing methods lack pulse width and length [8]. SFH 7051 is used for heart rate monitoring and SFH 750 for heart rate and oxygen content in the blood [9]. Fiber Bragg Grating (FBG) has greater immunity to electromagnetic interference with high sensitivity and conceivability. Accuracy of FBG is affected by temperature, crosstalk and motion artifacts [10]. Huotari et al. [11] projected Photoplethysmography (PPG) and Electro Mechanical Film (EMFi) sensors to estimate the arterial pulse wave. EMFi was used to measure the small arterial wall pressure and arterial elasticity [11]. Optimal method is a combination of Pressure sensor (Near Infrared) and photoelectric sensor array (9 pair photoelectric sensor, LED and photodiode) which improved the performance compared to traditional pulse diagnosis method. NIR light is absorbed by the blood and backscattered light is captured. Variations in backscattered light vary the blood flow in arteries. NIR is used measure tactile sensations of the physicians. Both temporal and spatial information is captured using an array. Multichannel optimization is carried out to improve the performance of the system. Optimization of Multichannel fusion was used to help detect diabetes and for classification of Cun, Guan and Chi.

Piezoelectric sensors: Piezoelectric sensors are active with good AC response at high frequencies. Mahesh et al. designed a polyvinylidene fluoride (PVDF) piezoelectric sensor for the measurement of pulse wave. Dynamic piezo film develops electrical charges proportional to the blood flow in radial artery. Vega and amplitude of the pulses are recorded by PVDF device [12]. Joshi et al. developed Nadi Tarangini for obtaining the dynamic pressure pulse of time series which elevated the time domain parameters such as pulse duration and pulse rate for different age groups. It is observed that, pulse duration increases and pulse rate decrease with the age [13]. Multifractality analysis explored self-similarity in arterial pulse and is used for diagnosis of skin disorder, aging and diabetes [14]. Kalange et al. proposed a system in which a transducer based on piezoelectric properties was used for detecting the human pulse. It was found that repetition frequency, amplitude and pulse shape of all three components are different [15].

Piezoresistive sensors: Piezoresistive sensor has a good dynamic and static response. Piezoresistive sensor exhibits change in the resistance for the applied of force/strain, pressure and acceleration. Piezo resistive sensor consists of a diaphragm that undergoes deformation which results in the change in resistance on applied pressure and which is measured using
Wheatstone bridge. Piezoresistive sensor has high sensitivity, accuracy and good response at low frequency, which is well suited for wrist pulse acquisition. Many Research groups captured the pulse using piezoresistive sensor (MPXM2053D) $[16,17]$. Kritika et al. developed single channel, real time monitoring system. Analysis of healthy states of the subject was carried out in frequency domain and classified into health and unhealthy states using various classifiers [16]. Wang et al. developed Multichannel fusion structure to capture pulse point, surrounding information of the pulse and to trap tactile sensations of the physicians. Multidimensional information gives accurate humans pathological condition [18]. Five different levels of hold -down pressure are measured to characterize the individual subject based on contour of the pulse. BMI of the subject is calculated based on Floating/ Sinking pulse patterns [19]. Comparing the performances of piezoelectric, ultrasonic and piezoresistive pressure sensors with accuracy, sensitivity and positioning, it can be concluded that piezoresistive is superior as it detects weak Kapha pulse amplitude. Pressure sensor array could be implemented with PVDF, PZT and piezoresistive, which can detect dynamic characteristics of components. Sensing area of the pressure sensor is large and could be used for computerized detection of pulses. Array of sensor were used to obtained temporal and spatial properties of a wrist pulse [20].

MEMS pressure sensors: Sun et al. developed a wearable device based on Micro Electro Mechanical System (MEMS) pressure sensors were used to detect the pulse wave velocity. Polydimethylsiloxane (PDMS) is packed around the MEMS sensor to avoid the environmental noise and to have efficient contact to the skin. Flexible PCB includes oxygen detector, accelerometer and three MEMS pressure sensors enclosed in a glove structured. Glove structure assists for accurate position of sensors when the subjects are at motion [21]. Zhichang et al. analyzed and observed the pulse contour of the pulse pressure which are correlated to the physiological state of cardiovascular and hypertension subjects [22]. The characteristics of a pulse shape defined with $\mathrm{K}$ value, lies between 0.32 and 0.38 for a healthy subject and given by where Pm, Ps and Pd is mean pressure, systolic pressure and diastolic pressure respectively. Pulse Wave Velocity is defined as whereas positioning of the MEMS sensors and as distance between the MEMS sensors positioning. Ching et al. developed Bi-sensing pulse diagnosis system to measure string like pulse patterns and observed elevate pattern in the hypertension subjects. Pulse depth and sensation of the pulse were recorded to correlate the parameters and to construct 3- Dimensional Pulse Map [22]. Bongrain et al. developed Ultra-thin flexible sensor based on piezoelectricity using Aluminum nitride (AIN) layer was used for monitoring micro deformations and also consumes very low power. Measuring system is used for monitoring of PWV during each cardiac cycle [23]. Thakker et al. designed wearable sensor for monitoring of PW V and provides compatible interface with wireless sensing system. Wearable sensor is compact, flexible, is highly sensitive to pressure, fast response and produces high stable output. Vital sensor shows both properties of ferroelectricity and 
piezoelectric responses. One sensor is attached to radial artery to measure the blood flow in the arties and another printed sensor is connected to communication amplifier for wireless detection of pulse. Maximum amplitudes of incident wave and reflected wave were used in the calculation of Augmentation Index (AI) of radial pulse [24].

\section{Literature Review}

Various methods are used to analyse and to extract the features from the acquired pulse waveform are time domain, frequency domain and nonlinear methods such as approximate entropy, sample entropy, correlation methods, and time frequency domains. Several hemodynamic parameters can be extracted from the pressure wave which determines health state of a subject.

\section{Time domain analysis}

Time domain analysis inspects on pulse shape and its contours. The pressure pulse consists of percussion wave, tidal wave and dichotic wave. Typical pulse are consists of peak amplitude, dicrotic notch and corresponding duration are the parameters used to analyze healthiness of the humans. In an unhealthy subject's tidal wave and dichotic wave amplitudes are very low. As static pressure increases, higher variations in pulse morphology were observed in unhealthy subjects compared to the healthy subjects [25]. Few research groups observed irregularities in the pulse waveform in the amplitudes and duration of tidal and dicrotic measured based on first order derivation [26]. Each pulse segment of a pulse waveform corresponds to onset of a pulse, peak amplitude, maximum positive slope, lowest negative slope, dicrotic notch and associated time intervals. Dynamic Time Wrapping (DTW), Euclidean Distance and Correlation coefficient methods were used to find similarity and dis-similarity index of pulse segment. Few research groups presented extraction of features of reflected wave of the peripherical arteries using first derivative of ensemble average method and observed DTW approach could be best to identify the Outlier line segment and to find similarity index in the pulse series [27]. Dimin Wang et al. observed variations in the width of a pulse indicated relaxed or tensed state of subjects [18]. Recurrence plot, Multi scale entropy and approximate entropy methods were applied to analyses pulse variabilities of a wrist pulse [28-33]. Bhaskar Thakker et al. had defined time domain pulse patterns for Gastritis and Arthritis based on Dicrotic Notch Suppression Ratio (DNSR) and BAD Notch Ratio [33].

The typical time domain analysis of a pulse waveform is shown in the Figure 4. Time domain parameter are Pulse amplitude (PP), Pulse time (PR), Rise Time (T1), Notch Amplitude (PDID), Notch Time (T2), Fall Time (T3), Width (W), Slope (PP/T1) and Pulse Transit Time (PTT). PP defined as distance between the onsets of the pulse point a1 to a2. PR defined as total pulse duration (t5-t1). Rise time defined as elapsed time between the start of the cycle and time of the pulse maximum. PDID defined as distance from the onset of the pulse point a1 to the notch minimum during the cycle a3.
Notch time defined as Elapsed time between the start of the cycle (t1) and the time of the notch minimum. Fall time represents elapsed time between the peak of the pulse (t2) and the time of the notch minimum (t3). Width is defined as width of the forward wave. Slope is defined as ratio of pulse amplitude to the rise time of the pulse.

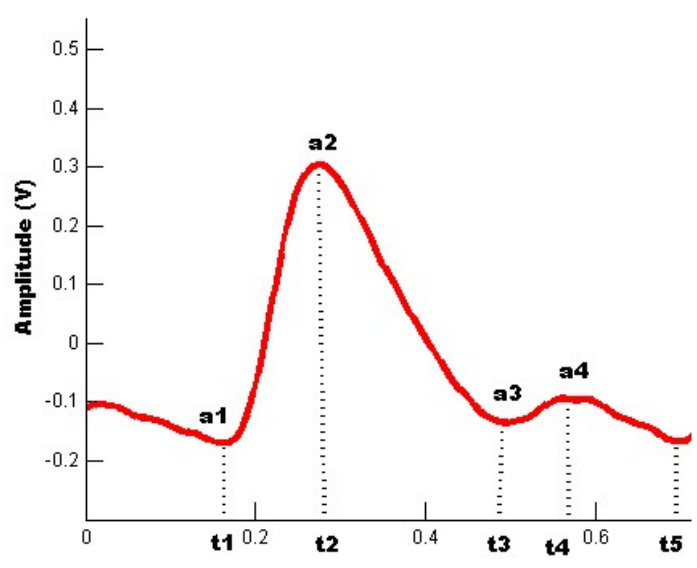

Figure 4. Time domain analysis

\section{Frequency domain analysis}

Frequency domain analysis is inspected on the resonant frequency, energy and spectrum of the pulse [20]. Different organs have different resonant frequencies and its harmonics which carries pathological and physiological information about an organ. Stiffness of the vessels is determined by deviation in the harmonic frequencies [34]. Power Spectrum could be computed using parametric methods and non-parametric methods. Parametric methods used to estimate the PSD are MA, ARMA and AR methods and non-parametric methods such as FFT, HHT DFT [32,35]. Several research groups have computed Band Energy Ratio (BER) for both heathy and unhealthy subject and reported the maximum energy distribution in the frequency band of $0-4 \mathrm{~Hz}$ for healthy subject and above $4 \mathrm{~Hz}$ for unhealthy subjects. Frequency domain features were trained to various classifiers to distinguish healthy and unhealthy subjects with a higher accuracy [17,32,36-39]. Kalanga et al. observed pulse repetition frequency for Vata, Pitta and Kapha were in range $1.35 \mathrm{~Hz}$ to $1.58 \mathrm{~Hz}, 1.16 \mathrm{~Hz}$ to $1.33 \mathrm{~Hz}$ and $0.833 \mathrm{~Hz}$ to $1.2 \mathrm{~Hz}$ respectively [15]. Few researchers have identified the presence of wiry, surging, choppy, slippery and normal pulses based on the frequency and phase shift components in the spectrum [36]. Mean and variance analysis is used to analyze abnormal pulse period helpful in diagnosis of cardiac disease [37-40].

Energy distribution is high in the lower band of frequencies $(0$ to $4 \mathrm{~Hz}$ ) for a normal subject as depicted in the Figure 5. Maximum energy is depicted in Pitta component at $\mathrm{Hz}$. 

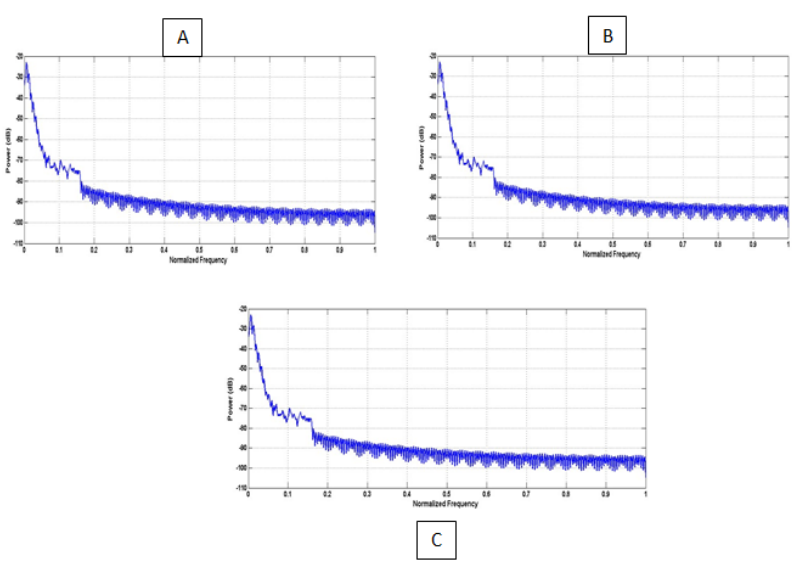

Figure 5. Power spectrum normal subject in (a) Vata, (b) Pitta, (c) Kapha pulses.

\section{Time-frequency analysis}

Time domain and Frequency domain analysis rely on the amplitude, frequency, covariance and spectral density of a signal which do not change with time. All the properties of the wrist pulse signal slowly change with time and then the signal is estimated by time-frequency method for accurate diagnosis of disease. Time -dependent frequency parameters are computed using Short Time Fourier Transform (STFT) and wavelet analysis for higher accuracy. Few researchers extracted time-frequency domain features using Gabor spectrogram and observed performance parameter mean square error. PSD of wrist pulse of various instants of time and frequency with different intensity levels computed by spectrogram for the identification of pregnant women and non- pregnant women [41]. Zhang et al. proposed Iterative Sliding Window Algorithm to eliminate the baseline wanders and for accurate estimation of valleys in the pulse with a sliding window. Period, value range, peak amplitude index were informative features of time domain and eight bands in a period represents frequency domain features which were used to detect lung cancer. Gaussian and gamma density function were used to analyses various TCM pulses based on the applied pressure [38].

\section{Discussion}

Evaluation of the vital information of the components depends exclusively upon the perception of the practitioner and varies from practitioner to practitioner based on his/her expertise or experience. No modern technique can determine the pathological condition of a subject. The paper presents a review of wrist pulse parameters for diseases like hypertension, diabetics, gastritis, Cancer and Inflammation and urinary tract infection disorders etc.

\section{Hypertension}

Hypertension is commonly known as Arterial Blood Pressure. In ancient days, hypertension was diagnosed by feeling the palpations on the wrist considering the Pulse Wave Velocity of pulse waveform [39-41]. Shu and Lee et al. examined and revealed that the Gwan pulse is highly associated with hypertension and found to be in frequency band of 0 to $13 \mathrm{~Hz}$. Classified healthy and hypertension subject by Naïve Bayes and Binary Logistic Regression algorithm [42,43]. Yan et al. observed that PPI (Maximum pulse amplitude in Gwan pulse of both left and right hand) parameter are highly associated with hypertension analyzed by Wavelet Packet Transform (WPT) and Hidden Markov Model (HMM) and observed that predictive power of right wrist is higher than left wrist pulse for hypertension subjects [44]. Li-jie Qiao et al. investigated that radial artery pulse Gwan wave variables were highly associated with arterial stiffness and hypertension. And observed time duration of all triplex (percussion, tidal, dicrotic notch) waves and amplitude of dicrotic wave decreases with an increase in the amplitude of main and tidal wave [45]. Mahesh et al. monitored of all the three pulses of non-cardiovascular subject and observed peak amplitudes of Vata, Pitta and Kapha are in the ratio of 4:2:1 respectively and observed vega of Pitta pulse is high throughout the day. Arterial Stiffness is a very important parameter in diagnosis of a cardiovascular disease [12]. Pooja More et al. measured parameters Stiffness Index (SI), Reflection Index (RI) of the Vata, Pitta and Kapha components and these parameters shown significance difference between healthy and unhealthy subjects using ANOVA test [46]. Franklin et al. projected pulse pressure is highly associated with hypertensive and Coronary heart diseases [47]. Research group measured pulse variability by nonlinear dynamic analysis called approximate entropy (ApEn) and observed that PSD of ApEn of a cardiovascular subject were much lower. Amount of variation in ApEn value used to distinguish various types cardiovascular disease and their physiological adaptabilities [30,31]. Wang et al. distinguished Rhythmic and Arrhythmic pulse based on Variation Range (VR) and Variation Coefficient (VC) of pulse interval of time series. Lempel-Ziv decomposition method used to distinguish different arrhythmic pulse patterns like Intermittent Pulse, Knotted Pulse or Running Pulse [48].

\section{Diabetes}

According to Ayurveda, variations (abnormal variations) in Vata, Pitta and Kapha components result in diabetics. Among the many diabetic patients, Ayurvedic practitioners observed excess pulse rates and lower peak amplitudes in the Kapha component. Arunkumar et al. computed Mean Approximate Entropy of Vata, Pitta and Kapha components of a diabetic subjects were less than 0.1771 [49]. Wang et al. [48] considered both periodic and non-periodic components (2-D) of the wrist pulse to analyze health status. Conventional features were extracted from periodic component and intraclass distribution parameters from the non- periodic space were used for accurate diagnosis of diabetes and pregnancy and it is observed that every fourth pulse of unhealthy subject in time series is highly correlated to healthy pulse pattern [7]. Pooja More et al. observed extra movement in Vata component of diabetic's subject [46-54]. 


\section{Gastrointestinal disorders}

According to Ayurvedic science Vata and Pitta components exhibit certain characteristics in subjects suffering from gastrointestinal disorders. Gastrointestinal disorders include indigestion, vomiting, liver expansion, liver cirrhosis, ulcerative colitis, piles, diarrhea etc. Bhaskar et al. examined Gastritis and Arthritis disorder based on irregularity and variation in deep pulse segment of Vata and Pitta elements. Strength of the pulse reduces as the increase in the static pressure applied results in elevation diastole phase on the next systolic phase into V shape, noticed as BAD Notch observed in unhealthy subject. Pulse Strength Ratio (PSR) was used to identify Deep and Moderate pulse. Observed variation of Vata pulse segment results in gastrointestinal disorders by sample entropy method. Pitta component of an unhealthy subject showed elevated energy in band of 8 to $10 \mathrm{~Hz}$ [55]. Energy concentration of a pulse was high in band of 2 to $6 \mathrm{~Hz}$ for after exercise and before lunch cases. Energy distribution was high in lower band of 0 to $2 \mathrm{~Hz}$ after the lunch case. Ratio of primary wave time of ascent to the signal length is more and less for after lunch and exercise cases respectively [54].

\section{Cancer}

Zhang et al. investigated the pulse waveform for lung cancer which was noticed in heart slow firing blood period. Features extracted from the heart slow firing blood period were used for identification of lung cancer. Observation were made on the morphology of pulse and concluded that Lung cancer subject have longer rapid ejection phase as compared to healthy subject [55]. Tawalare et al. presented the risk factors associated with breast cancer. Breast cancer occurs mostly in females and difficult to detect in its initial stage. Breast Cancer occurs mostly in Kapha prakriti type female individuals especially during menopause. Kapha Prakriti subject have lower metabolis $\mathrm{m}$ compared to subjects [28]. Zhang and his research team developed lung cancer monitoring system to provide service at home and for early detection. Recognition system was designed based on Jin's pulse Diagnosis (JPD) theory and 26 features were extracted both in time - frequency domain to classify lung cancer subject based on the pulse shape and energies in the band [38].

\section{Inflammation and urinary tract infection disorder}

In most of the liver problem patients, the researchers had observed the irregularity in Pitta component. Chow et al. diagnosed the of four types of inflammation such as Appendicitis, Acute Appendicitis, Pancreatitis, Duodenal Bulb Ulcer based on wrist pulse. Doppler parameters of cardiac cycle like peak systolic velocity (PSV), reverse velocity (RV), peak diastolic velocity (PDV), end diastolic velocity (EDV), duration of systole and duration of diastole were considered for analyses and were classified using SVM [50]. Lu et al. observed and analyzed liver disorder occurs due to irregularity in Kapha component. Subject encountered with liver problems showed weaker intensity above the 11th harmonic and the pulse could not be recorded. Experimental analysis was carried out to estimate phase of specific organs of both male and female [51-53]. Wang Nanyue et al. observed variations of time domain, frequency domain parameters of Zuoguan and Zuochi are used to identify the healthy subject or cirrhosis subject. Also able to distinguished Fatty Liver Disease (FLD) and cirrhosis subject depending upon the parameters of Youguan and Youchi [54-60]. Thakker et al. observed suppression of dicrotic notch at deep pressure in prostate enlargement urinary tract disorder which occurs in male subject [33].

\section{Conclusion}

In ancient India and China, uses wrist pulse analysis to diagnose health state of a subject in a cost effective and alternative way. Applying static pressure on radial artery to estimated dynamic pressure pulse of a subject. Analysis of dynamic pressure signal reflects various pathological conditions of a subject help to diagnosis the root cause of unhealthness.

\section{References}

1. Upadhyaya S. Vijnana N. Ancient pulse science. Chaukhamba Sanskrit Pratishthan, 2005.

2. Rhyner HH. Ayurveda. The gentle health system. Motilal Banarsidass, 2005.

3. Tiwari M. Ayurveda: Secrets of Healing. Motilal Books, 2001.

4. Lad V. Ayurveda: The science of self-healing: a practical guide. 2nd edn, Lotus Press, India, 1987.

5. Vasant L, Vasant DL. Secrets of the pulse the ancient art of ayurvedic pulse diagnosis. The Ayurvedic Press.

6. Yoon YZ, Lee MH, Soh KS. Pulse type classification by varying contact pressure. IEEE Engineering in Medicine and Biol Mag 2000; 19: 106-110.

7. Zhang D, Zuo W, Wang P. Generalized feature extraction for wrist pulse analysis: From 1-D time series to 2-D matrix. IEEE J Biomed Health Inform 21: 169-189.

8. Zhang H, Wu S, Li W, Wang Y, Dong M, Yang L. Precise detection of wrist pulse using digital speckle pattern interferometry. Evi-Based Compl Alter Med 2018; 7.

9. Goyal K, Agarwal R. Pulse based sensor design for wrist pulse signal analysis and health diagnosis. Biomed Res 2016; 28: 12.

10. Jia D, Chao J, Li S, Zhang H, Yan Y, Liu T, Sun Y. A fiber Bragg grating sensor for radial artery pulse waveform measurement. IEEE Transact Biomed Eng 2018; 65: 839-846.

11. Huotari M, Vehkaoja A, Määttä K, Kostamovaara J. Arterial pulse wave analysis based on PPG and EMFi measurements. 2012 13th Biennial Baltic Electronics Conference, Tallinn, 2012; 187-190.

12. Mahesh S, Manivannan M, Anandan T. Three radial artery pulse sensor design for Siddha based disease diagnosis. J Syst Cybern Inf 2008; 1: 19-23. 
13. Kulkarni JA, Chandran S, Jayaraman V, Kulkarni B. Nadi tarangini: A pulse based diagnostic system. Conf Proc IEEE Eng Med Biol Soc, Med. 2007: 2207-2210.

14. Joshi AJ, Chandran S, Jayaraman VK, Kulkarni BD. Multifractality in arterial pulse. Multifractality in arterial pulse 19th International Conference on Pattern Recognition, 2008; p. 1-4.

15. Kalange AE, Gangal SA. Piezoelectric sensor for human pulse detection. Defense Sci J 57: 109-114.

16. Goyal K, Agarwal R. Pulse based sensor design for wrist pulse signal analysis and health diagnosis. Biomed Res 2017; 28: 12.

17. Thakkar S, Thakker B. Wrist pulse acquisition and recording system. Communication on Applied Electronics. 1: $20-24$.

18. Wang D, Zhang D, Lu G. An optimal pulse system design by multichannel sensors. IEEE J Biomed Health Inform 2016; 20: 450-459.

19. Yoo SK, Shin KY, Lee TB, Jin SO. New pulse wave measurement method using different hold-down wrist pressures according to individual patient characteristics. Springerplus 2013; 27: 2-406.

20. Hu CS, Chung YF, Yeh CC, Luo CH. Temporal and spatial properties of arterial pulsation measurement using pressure sensor array. Evidence-Based Complementary and Alternative Medicine. 2012.

21. Sun Y, Dong Y, Gao R, Chu Y, Zhang M, Qian X, Wang X. Wearable pulse wave monitoring system based on MEMS sensors. Micromachines (Basel) 2018; 9: 90.

22.Zhichang L, Song Z, Werming Y. A research on characteristic information of pulse wave. J Beijing Polytech 1996; 3: 71-79.

23. Bongrain A, Rousseau L, Valbin L, Madaoui N, Lissorgues G, Verjus F, Chapon PA. A new technology of ultrathin AlN piezoelectric sensor for pulse wave measurement. Procedia Eng 2015 1; 120: 459-63.

24. Thakker B, Vyas AL. Outlier pulse detection and feature extraction for wrist pulse analysis: International Conference on Biological Science and Technologies (ICBST 2009), Norway.

25. Luo CH, Chung YF, Yeh CC, Si XC, Chang CC, Hu CS. Stringlike pulse quantification study by pulse wave in $3 \mathrm{D}$ pulse mapping. J Altern Complement Med 2012; 18: 924-31.

26. Sekine T, Sugano R, Tashiro T, Sato J, Takeda Y, Matsui H, Kumaki D, Dos Santos FD, Miyabo A, Tokito S. Fully Printed Wearable Vital Sensor for Human Pulse Rate Monitoring using Ferroelectric Polymer. Scientific reports 2018; 13: 8-4442.

27. Garg N, Kaur R, Garg H, Ryait HS, Kumar A. Wrist pulse signal features extraction: Virtual instrumentation. Adv Intell Syst 479: 135-142.

28. Tawalare k, Hedaoo G, Tawalare K. Prevalence of breast cancer in different personality traits (Prakriti). World J Pharm Pharmaceu Sci 2017; 6: 1653-1661.
29. Chung CY, Chung FY, Chu YW, Luo CH. Spatial feature extraction from wrist pulse signals. 1st International Conference on Orange Technologies Tainan, Taiwan.

30. Rangaprakash D, Dutt ND. Study of wrist pulse signals using time domain spatial features. Comp Elec Eng 2014; 45: 100-107.

31. Wang K, Xu L, Li Z, Zhang D, Li N, Wang S. Approximate entropy based pulse variability analysis. 16th IEEE Symposium Computer-Based Medical Systems, USA, 2003.

32. Yan J, Wang Y, Li F, Yan H, Xia C, Guo R. Analysis and classification of wrist pulse using sample entropy. IEEE International Symposium on IT in Medicine and Education, P.R. China, 2008.

33. Thakker B, Vyas AL. Suppressed dicrotic notch pulse classifier design. Intern J Machine Learning Comput 2011; $1: 2$.

34. Liu L, Li N, Zuo W, Zhang D, Zhang H. Multiscale sample entropy analysis of wrist pulse blood flow signal for disease diagnosis. International Conference on Intelligent Science and Intelligent Data Engineering, P.R. China, 2012.

35. Chang H, Chen J, Liu Y. Micro-piezoelectric pulse diagnose and frequency domain analysis of human pulse signals. J Trad Chinese Med Scie 2018; 5: 35-42.

36. Khaire NN, Joshi YV. Diagnosis of disease using wrist pulse signal for classification of pre-meal and post-meal samples. International Conference on Industrial Instrumentation and Control (ICIC). India, 2015.

37. Parikhrt K, Thakker B. Wrist pulse classification system for healthy and unhealthy subjects. Intern J Comp Appl 2015; 1: $120-124$.

38. Zhang Z, Zhang Y, Yao L, Song H, Kos A. A sensor-based wrist pulse signal processing and lung cancer recognition. J Biomed Inform 2018; 79: 107-116.

39. Thakker B, Vyasn AL. Wrist (pulse signal classification for health diagnosis. 4th International Conference on Biomedical Engineering and Informatics (BMEI), China.

40. Garg N, Babbar N. Feature extraction of wrist pulse signals using Gabor spectrogram. Indian J Sci Tech 2016; 9: 1-6.

41. Su ZY, Wang CC, Wu T, Wang YT, Tang FC. Instantaneous frequency-Time analysis of physiology signals: The application of pregnant Women's Radia 1 Artery Pulse Signals. Physica A: Statstical Mechanics and its Application 2008; 387: 485-494.

42. Shu JJ, Sun Y. Developing classification indices for Chinese pulse diagnosis. Complementary Therapies in Medicine 2007; 15: 190-198.

43. Lee BJ, Jeon YJ, Ku B, Kim JU, Bae JH, Kim JYI. Association of hypertension with physical factors of wrist pulse waves using a computational approach: A pilot study. BMC Complement Altern Med 2015; 15: 222.

44. Yan R, Zhou M, Sun W, Meng J. Analyzing wrist pulse signals measured with polyvinylidene fluoride film for hypertension identification. Sensors and Materials 2017; 29: 1339-1351. 
45. Qiao LJ, Qi Z, Tu LP, Zhang YH, Zhu LP, Xu JT, Zhang ZF. The association of radial artery pulse wave variables with the pulse wave velocity and echocardiographic parameters in hypertension. Evid-Based Compl Alt 2018; $1-11$.

46. More P, Joshi AJ, HR N. Developing a diagnostic tool for type 2 diabetes based on tridosha analysis through nadi pariksha. Int Ayurvedic Med J 2014; 2: 1100-1107.

47. Franklin SS, Khan SA, Wong ND, Larson MG, Levy D. Is pulse pressure useful in predicting risk for coronary heart disease? The Framingham Heart Study. Circulation 1999; 100: 354-360.

48. Wang L, Wang K, Xu LS. Lempe l-ziv decomposition based arrhythmic pulses recognition. Conf Proc IEEE Eng Med Biol Soc P.R. China, 2005.

49. Arunkumar N, Sirajudeen KM. Approximate Entropy based ayurvedic pulse diagnosis for diabetics-a case study 3rd International Conference on Trends in Information Sciences and Computing (TISC2011), 2011; 133-135.

50. Bisht A, Garg N, Ryait HS, Kumar A. Comparative analysis of DTW based outlier segregation algorithms for wrist pulse analysis. Indian J Sci Technol 2016; 9: 1-5.

51. Lu WA, Cheng CH, Wang YL, Wang WK. Pulse spectrum analysis of hospital patients with possible liver problems. Am J Chin Med 1996; 24: 315-320.

52. Thakker B, Lal Vyas A. Support vector machine for abnormal pulse classification. Int J Comput Appl T 2011; 22: $13-19$.

53. Xu LS, Wang KQ, Wang L, Li N. Pulse contour variability before and after exercise 19th IEEE Symposium on Computer-Based Medical Systems (CBMS'06), USA, 2006.

54. Nanyue W, Youhua Y, Dawei H, Bin X, Jia L, Tongda L, Liyuan X, Zengyu S, Yanping C, Jia W. Pulse diagnosis signals analysis of fatty liver disease and cirrhosis patients by using machine learning. Sci World J 2015; 9.

55. Kumar VGP, Deshpande S, Joshi A, More P, Nagendra. HR Significance of arterial stiffness in Tridosha analysis: A pilot study. J Ayurveda Integr Med 2017; 8: 252-256.

56. Thakker B, Lal Vyas A. Support vector machine for abnormal pulse classification. Int J Comput Appl T. 2011; 22: $13-9$.

57. Zhang Z, Zhuang X, Zhang Y, Kos A. Computerized Radia 1 Artery Pulse Signal Classification for Lung Cancer Detection 7th International Conference on Information Society and Technology, Serbia, 2007.

58. Chow WH, Chung KW, Tsang KF, Li BYS, Chui KT. Wrist pulse signal classification for inflammat ion of appendix, pancreas, and duodenum. IECON 2014 - 40th Annual Conference of the IEEE Industrial Electronics Society, USA, 2014.

59. Lee CT, Wei LY. Spectrum analysis of human pulse. IEEE Trans Biomed Eng 1983; 30: 348-352.

60. Veerabhadrappa ST, Vyas AL, Anand S. Changes in heart rate variability and pulse wave characteristics during normal pregnancy and postpartum. Int J Biomed Eng Tec 2015; 17: 99-114.

\section{*Correspondence to}

Suguna GC

Department of Electronics and Communication Engineering

JSS Academy of Technical Education

Bengaluru - 560060

India 